\title{
Harmonic analysis in value at risk calculations
}

\author{
Claudio Albanese and Luis Seco
}

\begin{abstract}
Value at Risk is a measure of risk exposure of a portfolio and is defined as the maximum possible loss in a certain time frame, typically 1-20 days, and within a certain confidence, typically $95 \%$. Full valuation of a portfolio under a large number of scenarios is a lengthy process. To speed it up, one can make use of the total delta vector and the total gamma matrix of a portfolio and compute a Gaussian integral over a region bounded by a quadric. We use methods from harmonic analysis to find approximate analytic formulas for the Value at Risk as a function of time and of the confidence level. In this framework, the calculation is reduced to the problem of evaluating linear algebra invariants such as traces of products of matrices, which arise from a Feynmann expansion. The use of Fourier transforms is crucial to resum the expansions and to obtain formulas that smoothly interpolate between low and large confidence levels, as well as between short and long time horizons.
\end{abstract}

\section{Introduction.}

The notion of Value at Risk (VaR), introduced in the J. P. Morgan RiskMetrics document [JPM], captures the risk exposure of a portfolio in terms of the largest possible loss within a certain confidence interval.

In the RiskMetrics framework, one deals with portfolios subject to a number of risk factors whose evolution is a geometric Brownian motion with a given covariance matrix. The full valuation method 
consists of repricing the portfolio under a number of scenarios by calling all the relevant pricing functions. This procedure is computationally very intensive. In typical applications with portfolios that consist of several hundred thousand instruments, not more than a few thousand scenarios can be priced overnight with current technologies. The small number of scenarios results in large inaccuracies in the Value at Risk measurement. The use of rather unsophisticated pricing models can speed up the calculation but is also at the origin of uncontrollable errors.

An alternative that has been advocated in the RiskMetrics technical document is to use the quadratic approximation for the portfolio variation as a function of the underlying risk factors. To obtain this representation, the knowledge of the total delta vector and of the total gamma matrix is required. This leads to the problem of evaluating an integral of the form

$$
I_{0}(K)=\int_{\Delta x+(1 / 2)(x, \Gamma x) \leq K} \exp (-\pi(x, \mathbb{A} x)),
$$

for certain vectors $\Delta$ and matrix $\Gamma$. To our knowledge, the problem of estimating (1.1) was first considered by Ruben [Rub] (in the case of positive definite $\Gamma$ and zero $\Delta$ ), and then extended by a number of other authors, see $[\mathrm{KJB}]$ and references therein. An asymptotic expansion in the large confidence limit has been obtained by Quintanilla (see [Q]).

The notion of Value at Risk owes its popularity to the fact that it captures, with just one parameter of intuitive meaning, the risk exposure of a portfolio. However, the Value at Risk evolves with time and is subject to stochastic fluctuations which reflect the evolution of the risk factors and the evolution of the composition of the portfolio itself. The sensitivity of the Value at Risk with respect to the dynamics of the underlying risk factors depends on the relative importance of delta and gamma risks. To capture this effect, it is useful to use dual variables which give the sensitivity to the total delta and the total gamma risk of a given portfolio.

In our setting, duality transformations involve Fourier transforms. After an initial simultaneous diagonalization of the covariance matrix $\mathbb{A}$ and of the total gamma of the portfolio, we reduce the calculation to an integral of a Gaussian over a high dimensional quadric. This integral is computed using techniques from harmonic analysis, which reduce all calculations to the Fourier transform of quadrics. In the case of positive definite Gammas, this reduces to explicit Bessel functions; the general case is not much more difficult. The Paley-Wiener theorem guarantees 
that our formulas have adequate computational properties. This result can be strengthened by deriving analytic formulas which give the asymptotic behaviour and determine the relevant Fourier transforms up to smooth multipliers. The moments of these transforms can be computed by means of a technique related to Feynmann diagrams. This gives rise to matrix invariants such as traces and determinants, which yield analytic formulas for the Value at Risk as a function of the time horizon and of the confidence level. In this context, the use of Fourier transforms is crucial to resum the expansions and to obtain formulas that smoothly interpolate between low and large confidence levels, as well as between short and long time horizons.

By changing coordinates, the integral in (1.1) can be reduced to a convolution of integrals of the same form but with a positive $\Gamma$. In this case, Fourier transforms give rise to the two following representations: first,

$$
\begin{aligned}
I_{0}(K(R))=R^{n / 2}( & \int_{-\infty}^{0} \frac{J_{n / 2}(2 R \pi \sqrt{|\widehat{\alpha}|}) G(\widehat{\alpha})}{\left(2|\widehat{\alpha}|^{n / 4}\right)} d \widehat{\alpha} \\
& +\pi i \int_{-\infty}^{0} \int_{\widehat{\alpha} \tan ^{-1}\|v\|^{2}}^{0} \frac{J_{n / 2}(2 R \pi \sqrt{|\widehat{\alpha}|}) F(\widehat{\alpha}, \widehat{\beta})}{(2|\widehat{\alpha}|)^{n / 4}} \\
& \left.\cdot \frac{\cos (2 \pi \sqrt{2 \widehat{\beta}})-1}{2 \pi^{2} \widehat{\beta}} d \widehat{\alpha} d \widehat{\beta}\right),
\end{aligned}
$$

for a certain function $K(R)$ and for suitable functions $G$ and $F$, which arise as Fourier transforms of certain determinant functions. The integer $n$ represents the number of risk factors (or underlyings) in the portfolio under consideration.

In this expression, the first term corresponds to the VaR of a perfectly $\delta$-hedged portfolio, while the second captures the VaR of hedging imperfections. In fact, we will also obtain a second expression, as an asymptotic expansion of the form

$$
I_{0}(K(R))=R^{n / 2} \sum_{j=0}^{\infty} \frac{1}{j !} \int_{-\infty}^{0} \frac{J_{n / 2}(2 R \pi \sqrt{|\widehat{\alpha}|})}{(2|\widehat{\alpha}|)^{n / 4}} H^{(j)}(\widehat{\alpha}) d \widehat{\alpha}
$$

for suitable functions $H^{(j)}$. Each term in this expansion corresponds to increasing degrees of delta-hedge slippage. It is obtained by expanding the first in powers of the $\Delta$ vector and is convenient in the limit of 
small $\Delta$ or of large time horizon. The functions $G(\widehat{\alpha}), F(\widehat{\alpha}, \widehat{\beta})$ and $H^{(j)}(\widehat{\alpha})$ admit an integral representation that allows one to find their asymptotic behaviour at the boundaries of their support. Moreover, the Fourier transform of these functions can be computed explicitly and the moments admit an expansion in Feynmann diagrams. This expansion can be used jointly with the asymptotic analysis to find an approximation scheme to efficiently interpolate between large and small values of the arguments based on the knowledge of linear invariants of the matrix $D$ and the vector $v$, such as $\operatorname{Tr} D^{k},\|v\|$ and $\left(v, D^{k} v\right)$.

The interest of analytical formulas for VaR of the type presented in this paper is manifold, and not unrelated to the interest of analytical expressions for traditional pricing theories. First, they allow for further analysis and calibration for different portfolio parameters. Second, they allow for VaR calibration techniques based on historical P\&L data.

This article is organized into seven sections. In the next one, we present the general framework and the main formulas in our analysis. In the third, fourth and fifth sections, which are rather technical, we provide all the details that justify our approach and the formulas it gives rise to. Based on these results, in the sixth section we derive an efficient approximation scheme for value at risk calculations. The last section contains concluding remarks.

\section{Value at Risk.}

Consider a portfolio of price $\Pi$ consisting of a combination of underlying securities $S_{j}$, for $j=1, \ldots, n$, which we assume to be $\log$ normally distributed with covariance matrix $\mathbb{V}$. The Value at Risk of the portfolio is defined to be the number $K$ such that

$$
\operatorname{Prob}(\Pi(0)-\Pi(t)) \geq K=\varepsilon,
$$

where $\varepsilon$ is a small number (typically 0.05 ), and $t$ is a small time window (i.e., 1 day).

In this paper we consider only portfolios that are smooth over time horizons of interest. This includes most traded securities with some exceptions as, for instance, barrier options when the price of the underlying is near the barrier. To apply our methods, a split of the portfolio into a regular and a singular sub-portfolio is necessary if such singular securities are present. 
To leading order in time, we approximate the value of the portfolio by today's deltas and gammas,

$$
\Delta_{0}=\nabla_{\mathbf{S}} \Pi=\left(\frac{\partial \Pi}{\partial S_{1}}, \ldots, \frac{\partial \Pi}{\partial S_{n}}\right),
$$

and

$$
\Gamma_{0}=\operatorname{Hess}_{\mathbf{s}} \Pi=\left\{\frac{\partial^{2} \Pi}{\partial S_{i} \partial S_{j}}\right\},
$$

in the sense that, in the near future $t$,

$$
\Pi(t) \approx \Pi(0)+\Delta_{0} \cdot(\mathbf{S}(t)-\mathbf{S}(0))+\frac{1}{2}(\mathbf{S}(t)-\mathbf{S}(0)) \cdot \Gamma_{0} \cdot(\mathbf{S}(t)-\mathbf{S}(0))^{\dagger}
$$

Our assumption on log-normality means that

$$
\mathbf{S}(t)=\left(S_{1}(0) e^{\eta_{1}}, \ldots, S_{n}(0) e^{\eta_{n}}\right),
$$

with $E=\left(\eta_{1}, \ldots, \eta_{n}\right)$ a normally distributed random vector,

$$
\operatorname{Prob}\{E \in \Omega\}=\pi^{-n / 2} \int_{\Omega} e^{-(x-m) \mathbb{V}^{-1}(x-m)^{t}} \frac{d x}{\sqrt{\operatorname{det} \mathbb{V}}} .
$$

Taylor-expanding the exponential, (2.2) becomes

$$
\begin{aligned}
\Pi(t)= & \Pi(0)+\sum_{i=1}^{n} S_{i}(0) \Delta_{0}^{i}\left(\eta_{i}+\frac{1}{2} \eta_{i}^{2}\right) \\
& +\frac{1}{2} \sum_{i, j} S_{i}(0) S_{j}(0) \eta_{i} \eta_{j} \Gamma_{0}^{i, j}+\mathcal{O}\left(|E|^{3}\right) \\
= & \Pi(0)+\Delta \cdot E+\frac{1}{2} E \cdot \Gamma \cdot E^{t}
\end{aligned}
$$

where

$$
\Delta_{i}=S_{i}(0) \cdot \Delta_{0}^{i}, \quad \Gamma_{i, j}= \begin{cases}S_{i}(0) S_{j}(0) \Gamma_{0}^{i, j}, & \text { if } i \neq j, \\ S_{i}^{2}(0) \Gamma_{0}^{i, i}+\Delta_{i}, & \text { if } i=j .\end{cases}
$$

According to this approximation, the Value at Risk $K$ of the portfolio (2.1) can be approximated by the number $K^{\prime}$ such that

$$
\operatorname{Prob}\left\{\Delta \cdot E+\frac{1}{2} E \cdot \Gamma \cdot E^{t} \leq-K^{\prime}\right\}=\varepsilon,
$$


which, using (2.3), becomes

$$
\pi^{-n / 2} \int_{x \cdot \Delta+(1 / 2) x \Gamma x^{\dagger} \leq-K^{\prime}} e^{-(x-m) \mathbb{V}^{-1}(x-m)^{t}} \frac{d x}{\sqrt{\operatorname{det} V}}=\varepsilon,
$$

or

$$
\pi^{-n / 2} \int_{x \cdot \Delta^{\prime}+(1 / 2) x \Gamma x^{\dagger} \leq K^{\prime \prime}} e^{-x \mathbb{V}^{-1} x^{t}} \frac{d x}{\sqrt{\operatorname{det} \mathbb{V}}}=\varepsilon
$$

for

$$
\begin{gathered}
\Delta^{\prime}=\Delta+m \Gamma \\
K^{\prime \prime}=-K^{\prime}-m \cdot \Delta-\frac{1}{2} m \Gamma m^{t} .
\end{gathered}
$$

Hence, the goal of this paper is to produce an efficient scheme to compute multidimensional integrals of the type

$$
I_{0}(K)=\int_{x \cdot \Delta+(1 / 2)(x, \Gamma x) \leq K} e^{-\pi(x, \mathbb{A} x)} d x
$$

Here, $\mathbb{A}$ is a symmetric positive definite matrix, while $\Gamma$ is just symmetric. We shall assume that $\Gamma$ is non-singular as this is the generic case; the singular case can be reduced to this, plus explicit erf terms.

\section{Diagonalizations.}

Lemma 1. We have that

$$
\sqrt{\operatorname{det} \mathbb{A}} I_{0}(K)=1-\sqrt{\operatorname{det}\left(\frac{\mathbb{A}}{|\Gamma|}\right)} I(\bar{K}),
$$

where

$$
I(\bar{K})=\int_{\left|x_{+}\right|^{2}-\left|x_{-}\right|^{2} \leq \bar{K}} e^{-\pi((x-v), \mathbb{D}(x-v))} d x,
$$

with $\bar{K}, v$ and $\mathbb{D}$ defined below.

Proof. The matrices $\mathbb{A}$ and $\Gamma$ are as follows. $\mathbb{A}$ is symmetric positive definite, with diagonal form given by

$$
\mathbb{A}=\mathbb{Q}^{-1} \mathbb{M} \mathbb{Q}
$$


where $\mathbb{Q}$ is orthogonal and

$$
\mathbb{M}=\left(\begin{array}{lll}
a_{1} & & \\
& \ddots & \\
& & a_{n}
\end{array}\right) .
$$

We have that

$$
(x, \mathbb{A} x)=(\mathbb{Q} x, \mathbb{M} \mathbb{Q} x) .
$$

$\Gamma$ is symmetric non-singular. The matrix and the vector

$$
\Gamma^{\prime}=\mathbb{M}^{-1 / 2} \mathbb{Q} \Gamma \mathbb{Q}^{-1} \mathbb{M}^{-1 / 2}, \quad \Delta^{\prime}=\mathbb{M}^{-1 / 2} \mathbb{Q}^{-1} \Delta
$$

are such that

$$
(x, \Gamma x)=\left(\mathbb{M}^{1 / 2} \mathbb{Q} x, \Gamma^{\prime} \mathbb{M}^{1 / 2} \mathbb{Q} x\right), \quad \Delta \cdot x=\Delta^{\prime} \mathbb{M}^{1 / 2} \mathbb{Q} x .
$$

In terms of these matrices, the integral in (2.6) is given by

$$
I_{0}(K)=\frac{1}{\sqrt{\operatorname{det} \mathbb{A}}} \int_{\Delta^{\prime} x+(1 / 2)\left(x, \Gamma^{\prime} x\right) \leq K} \exp \left(-\pi\|x\|^{2}\right) d x .
$$

Let $\mathbb{S}$ be the orthogonal transformation which diagonalizes $\Gamma^{\prime}$, i.e.

$$
\Gamma^{\prime}=\mathbb{S}^{-1} \mathbb{L} \mathbb{S}
$$

and let $\Delta^{\prime \prime}, \mathbb{D}$ be such that

$$
(x, x)=(\sqrt{|\mathbb{L}|} \mathbb{S} x, \mathbb{D} \sqrt{|\mathbb{L}|} \mathbb{S} x), \quad \Delta^{\prime} \cdot x=\Delta^{\prime \prime} \sqrt{|\mathbb{L}|} \mathbb{S} x,
$$

i.e.

$$
\mathbb{D}=|\mathbb{L}|^{-1}, \quad \Delta^{\prime \prime}=|\mathbb{L}|^{-1 / 2} \mathbb{S}^{-1} \Delta^{\prime}=|\mathbb{L}|^{-1 / 2} \mathbb{S}^{-1} \mathbb{M}^{-1 / 2} \mathbb{Q}^{-1} \Delta
$$

We have that

$$
I_{0}(K)=\frac{1}{\sqrt{\operatorname{det} \Gamma}} \int_{\Delta^{\prime \prime} \cdot x+(x,(\mathbb{L} /|\mathbb{L}|) x) \leq K} \exp (-\pi(x, \mathbb{D} x)) d x .
$$

Setting

$$
v=\frac{1}{2} \frac{\mathbb{L}}{|\mathbb{L}|} \Delta^{\prime \prime}, \quad \bar{K}=K-(v, v)+\Delta^{\prime \prime} v,
$$


and shifting coordinates, we arrive at an integral of the form

$$
I_{0}(K)=\frac{1}{\sqrt{\operatorname{det} \Gamma}} \int_{\left|x_{+}\right|^{2}-\left|x_{-}\right|^{2} \leq \bar{K}} \exp (-\pi(x-v, \mathbb{D}(x-v))) d x .
$$

where the vector $x=\left(x_{-}, x_{+}\right)$is split into the components along the eigenspaces of $\mathbb{L} /|\mathbb{L}|$.

\section{The positive definite case.}

The goal is to compute the integral

$$
I\left(R^{2}\right)=\int_{|x|^{2} \leq R^{2}} e^{-\pi(x-v) \mathbb{D}(x-v)^{t}} d x,
$$

when $\mathbb{D}$ is an $d \times d$ positive definite matrix. We denote its (positive) diagonal elements by $\gamma_{j}, j=1, \ldots, d$.

The expansion of the Bessel function

$$
\frac{J_{d / 2}(2 z)}{|z|^{d / 2}} \cos (2 w)=\sum_{k, j} a_{k, j} z^{2 k} w^{2 j} .
$$

has coefficients

$$
a_{k, j}=\frac{(-1)^{k+j} 2^{2 j}}{k !(2 j) ! \Gamma\left(k+1+\frac{d}{2}\right)} .
$$

Note that we have

$$
\sum_{k, j}\left|a_{k, j}\right| z^{2 k} w^{2 j}=\frac{I_{d / 2}(2 z)}{|z|^{d / 2}} \cosh (2 w),
$$

where $I_{n / 2}$ denotes the modified Bessel function. We will also need the related hypergometric functions

$$
\mathcal{B}(z, w)=\sum_{k, j}\left|a_{k, j}\right| z^{k} w^{j}=\frac{\mathcal{Z}_{d / 2}(2 z)}{|z|^{d / 4}} \mathcal{C}(2 w),
$$

where

$$
\begin{gathered}
\mathcal{Z}_{d / 2}(2 z)= \begin{cases}I_{d / 2}(\sqrt{2 z}), & \text { if } z>0, \\
J_{d / 2}(\sqrt{2|z|}), & \text { if } z<0,\end{cases} \\
\mathcal{C}(2 z)= \begin{cases}\cosh (2 \sqrt{z}) & \text { if } z>0, \\
\cos (2 \sqrt{|z|}) & \text { if } z<0,\end{cases}
\end{gathered}
$$


and

$$
\widetilde{\mathcal{B}}(z, w)=\sum_{k, j}\left|a_{k, j+1}\right| z^{k} w^{j}=\frac{\mathcal{Z}_{d / 2}(2 z)}{|z|^{\frac{d}{4}}} \frac{\mathcal{C}(2 w)-1}{w} .
$$

The only property we will use for these functions is that they are bounded for negative arguments and grow at most exponentially for positive arguments.

Lemma 2. Define

$$
\begin{gathered}
\mathbb{N}(\alpha, \beta)=\mathbf{1}+i \alpha \mathbb{D}+i \beta \mathbb{D}^{1 / 2} v^{t} v \mathbb{D}^{1 / 2} \\
f(\alpha, \beta)=\int_{\mathbb{R}^{d}} e^{-\pi \xi \mathbb{N}(\alpha, \beta) \xi^{t}} d \xi=(\operatorname{det} \mathbb{N}(\alpha, \beta))^{-1 / 2} .
\end{gathered}
$$

We have

$$
I\left(R^{2}\right)=\left.\left.\pi^{d / 2} \sum_{k, j=0} R^{n+2 k}(\pi i)^{k+j} a_{k j} \frac{\partial^{k}}{\partial \alpha^{k}}\right|_{\alpha=0} \frac{\partial^{j}}{\partial \beta^{j}}\right|_{\beta=0} f(\alpha, \beta) .
$$

The Fourier transform of the characteristic function of a ball is a Bessel function. Therefore, using Parseval's identity, in dimension $n$ we have

$$
\begin{aligned}
I\left(R^{2}\right) & =\int_{|x| \leq R} e^{-\pi(x-v) \mathbb{D}(x-v)^{t}} d x \\
& =R^{d} \int_{\mathbb{R}^{d}} \frac{J_{d / 2}(2 \pi R|\xi|)}{|R \xi|^{d / 2}} e^{-\pi\left(\xi, \mathbb{D}^{-1} \xi\right)} \cos (2 \pi \xi \cdot v) \frac{d \xi}{\sqrt{\operatorname{det} \pi \mathbb{D}}} \\
& =\sum_{k, j}^{\infty} R^{d+2 k} \pi^{k+j} a_{k j} \int_{\mathbb{R}^{d}}|\xi|^{2 k}(\xi \cdot v)^{2 j} e^{-\pi \xi \mathbb{D}^{-1} \xi} \frac{d \xi}{\sqrt{\operatorname{det} \pi \mathbb{D}}} .
\end{aligned}
$$

Since

$$
(v \cdot \xi)^{2}=\xi^{t} v^{t} v \xi,
$$

we have that

$$
\begin{aligned}
\int_{\mathbb{R}^{d}}|\xi|^{2 k}(\xi \cdot v)^{2 j} e^{-\pi \xi \mathbb{D}^{-1} \xi} d \xi & \\
& =\left.\left.(-i)^{-k-j} \frac{\partial^{k}}{\partial \alpha^{k}}\right|_{\alpha=0} \frac{\partial^{j}}{\partial \beta^{j}}\right|_{\beta=0} \int e^{-\pi \xi\left(\mathbb{D}^{-1}+i \alpha+i \beta v^{t} v\right) \xi} d \xi \\
& =\left.\left.\pi^{d / 2} i^{k+j} \frac{\partial^{k}}{\partial \alpha^{k}}\right|_{\alpha=0} \frac{\partial^{j}}{\partial \beta^{j}}\right|_{\beta=0}\left(\operatorname{det}\left(\mathbb{D}^{-1}+i \alpha+i \beta v^{t} v\right)\right)^{-1 / 2} .
\end{aligned}
$$


The function $f$ is smooth since the real part of $\mathbb{N}$ is positive. $f$ can easily be computed using the following elementary result in linear algebra.

Lemma 3. Let $\gamma_{j}>0$ be the eigenvalues of $\mathbb{D}$, for $j=1, \ldots, d$, and $v \in \mathbb{R}^{d}$. Then, for $\alpha, \beta \in \mathbb{C}$ we have

$\operatorname{det}\left(1+\alpha \mathbb{D}+\beta \mathbb{D}^{1 / 2} v^{t} v \mathbb{D}^{1 / 2}\right)=\left(\prod_{j=1}^{d}\left(1+\alpha \gamma_{j}\right)\right)\left(1+\sum_{j=1}^{d} \frac{\beta \gamma_{j}\left|v_{j}\right|^{2}}{1+\alpha \gamma_{j}}\right)$.

Proof. For $w \in \mathbb{R}^{d}$, we have

$$
\operatorname{det}\left(\mathbf{1}+w^{t} w\right)=1+|w|^{2} .
$$

This follows simply by rotating $v$ with a unitary $\mathbb{U}$ so it is of the form $(|w|, 0, \ldots, 0)$, for which the claim is obvious.

We find

$$
\begin{aligned}
& \operatorname{det}\left(\mathbf{1}+\alpha \mathbb{D}+\beta \mathbb{D}^{1 / 2} v^{t} v \mathbb{D}^{1 / 2}\right) \\
& =\operatorname{det}(\mathbf{1}+\alpha \mathbb{D}) \operatorname{det}\left(\mathbf{1}+\beta(\mathbf{1}+\alpha \mathbb{D})^{-1 / 2} \mathbb{D}^{1 / 2} v^{t} v \mathbb{D}^{1 / 2}(\mathbf{1}+\alpha \mathbb{D})^{-1 / 2}\right) \\
& =\left(\prod_{j=1}^{d}\left(1+\alpha \gamma_{j}\right)\right)\left(1+\sum_{j=1}^{d} \frac{\beta \gamma_{j}\left|v_{j}\right|^{2}}{\left(1+\alpha \gamma_{j}\right)}\right) .
\end{aligned}
$$

Lemma 4. The function $f(\alpha, \beta)$ can be extended as an analytic function to the domain

$$
\mathcal{Q}=\left\{(\alpha, \beta) \in \mathbb{C}^{2}: \operatorname{Im} \alpha \max \gamma_{j}+\operatorname{Im} \beta v D v^{t}<1\right\} .
$$

Proof. Modify (4.2) to

$$
f(\alpha, \beta)=\int_{\mathbb{R}^{d}} \exp \left(-\pi \xi \cdot\left(\mathbf{1}+i \alpha \mathbb{D}+i \beta \mathbb{D}^{1 / 2} v^{t} v \mathbb{D}^{1 / 2}\right) \cdot \xi^{t}\right) d \xi
$$

for complex $\alpha$ and $\beta$. Since

$$
\xi \mathbb{D} \xi^{t} \leq|\xi|^{2} \max _{j} \gamma_{j}, \quad \xi \mathbb{D}^{1 / 2} v^{t} v \mathbb{D}^{1 / 2} \xi^{t} \leq|\xi|^{2}\left(v \mathbb{D} v^{t}\right)
$$

we see that the integral defining $f$ in (4.3) is absolutely convergent for all $\alpha$ and $\beta$ in $\mathcal{Q}$. 


\section{Lemma 5.}

$$
|f(\alpha, \beta)| \leq|\operatorname{Re} \alpha|^{-(d-1) / 2}(\operatorname{det} \mathbb{D})^{-1 / 2}\left(|\operatorname{Re} \alpha|+|\operatorname{Re} \beta||v|^{2}\right)^{-1 / 2},
$$

where $(\alpha, \beta) \in \mathcal{D}$.

Proof. Let $\mathbb{A}$ and $\mathbb{B}$ be positive definite matrices.

$$
\int_{\mathbb{R}^{d}} e^{-\pi \xi \mathbb{A} \xi^{t}} e^{\pi i \xi \mathbb{B} \xi^{t}} d \xi=\frac{e^{\pi i d / 4}}{\sqrt{\operatorname{det}(\mathbb{A} \mathbb{B})}} \int_{\mathbb{R}^{d}} e^{-\pi \xi \mathbb{A}^{-1} \xi^{t}} e^{\pi i \xi \mathbb{B}^{-1} \xi^{t}} d \xi .
$$

Hence,

$$
\begin{aligned}
\left|\int_{\mathbb{R}^{d}} e^{-\pi \xi \mathbb{A} \xi^{t}} e^{\pi i \xi \mathbb{B} \xi^{t}} d \xi\right| & \leq(\operatorname{det}(\mathbb{A} \mathbb{B}))^{-1 / 2} \int_{\mathbb{R}^{d}} e^{-\pi \xi \mathbb{A}^{-1} \xi^{t}} d x \\
& =(\operatorname{det} \mathbb{B})^{-1 / 2}
\end{aligned}
$$

We apply this to (4.3) with

$$
\begin{gathered}
\mathbb{A}=1-(\operatorname{Im} \alpha) \mathbb{D}-(\operatorname{Im} \beta) v^{t} v, \\
\mathbb{B}=\sigma \mathbb{D}+t v^{t} v, \\
\sigma=\operatorname{Re} \alpha, t=\operatorname{Re} \beta,
\end{gathered}
$$

to obtain

$$
\begin{aligned}
|f(\alpha, \beta)| & \leq\left(\operatorname{det}\left(|\sigma| \mathbb{D}+|t| v^{t} v \mathbb{D}\right)\right)^{-1 / 2} \\
& =|\sigma|^{-d / 2}(\operatorname{det} \mathbb{D})^{-1 / 2} \operatorname{det}\left(1+\frac{|t|}{|\sigma|} v^{t} v\right)^{-1 / 2}
\end{aligned}
$$

We use Lemma 3 to compute the last determinant above and conclude that

$$
|f(\alpha, \beta)| \leq|\sigma|^{-d / 2}(\operatorname{det} \mathbb{D})^{-1 / 2}\left(1+\frac{|t|}{|\sigma|}|v|^{2}\right)^{-1 / 2},
$$

as claimed.

It can easily be seen using stationary phase estimates that the bound in the lemma above is sharp. An immediate consequence of this result is that the function $f$ is integrable in $\alpha$ but not in $\beta$. 
Lemma 6. Fix constants $T<\|\mathbb{D}\|^{-1}$, and $U, V$ such that $U\|\mathbb{D}\|+$ $V v \mathbb{D} v^{t}<1$. then,

$$
\int_{-\infty}^{\infty}|f(\sigma+i T, 0)| d \sigma<\infty, \quad \int_{-\infty}^{\infty} \int_{-\infty}^{\infty}\left|\frac{\partial f}{\partial \beta}(\sigma+i U, t+i V)\right| d \sigma d t<\infty
$$

Proof. It is enough to establish the integrability of $f$ and $f_{\beta}$ at infinity, since they are bounded inside $\mathcal{D}$. Thus the first bound follows from Lemma 5 . For the second, just note that by Lemma 3 the determinant function is linear in $\beta$. Hence,

$$
\begin{aligned}
\frac{\partial f}{\partial \beta} & =-\frac{i}{2} f^{3}(\alpha, \beta) \prod_{j=1}^{d}\left(1+i \alpha \gamma_{j}\right) \sum_{j=1}^{d} \frac{\gamma_{j}\left|v_{j}\right|^{2}}{1+i \alpha \gamma_{j}} \\
& =\frac{i}{2} \prod_{j=1}^{d}\left(1+i \alpha \gamma_{j}\right)^{-1 / 2} \frac{\sum_{j=1}^{d} \frac{\gamma_{j}\left|v_{j}\right|^{2}}{1+i \alpha \gamma_{j}}}{\left(1+i \beta \sum_{j=1}^{d} \frac{\gamma_{j}\left|v_{j}\right|^{2}}{1+i \alpha \gamma_{j}}\right)^{3 / 2}}
\end{aligned}
$$

Therefore,

$$
\begin{aligned}
\left|\frac{\partial f}{\partial \beta}\right| & \leq \frac{|f|^{3} v D v^{t}}{2 \min \left|1+i \alpha \gamma_{j}\right|} \prod_{j=1}^{d}\left|1+i \alpha \gamma_{j}\right| \\
& \leq \frac{1}{2}|f|^{3} v D v^{t} \max \left|1+i \alpha \gamma_{j}\right|^{d-1} \\
& \leq \frac{v D v^{t}}{2(\operatorname{det} D)^{3 / 2}} \frac{\max \left|1+i \alpha \gamma_{j}\right|^{d-1}}{|\sigma|^{3(d-1) / 2}\left(|\sigma|+|t||v|^{2}\right)^{3 / 2}}
\end{aligned}
$$

which is clearly integrable at infinity.

Our next target is to rewrite Lemma 2 using the Fourier transform of $f$. Define

$$
\begin{gathered}
F(\widehat{\alpha}, \widehat{\beta})=\int_{\mathbb{R}^{2}} e^{-2 \pi i(\widehat{\alpha} \alpha+\widehat{\beta} \beta)} \frac{\partial f}{\partial \beta}(\alpha, \beta) d \alpha d \beta, \\
G(\widehat{\alpha})=\int_{\mathbb{R}} e^{-2 \pi i \widehat{\alpha} \alpha} f(\alpha, 0) d \alpha .
\end{gathered}
$$


Both are well defined due to Lemma 6 .

Lemma 7 (Paley-Wiener). Let $\varepsilon>0$.

$$
|F(\widehat{\alpha}, \widehat{\beta})| \leq C_{\varepsilon} e^{-2 \pi\left(c_{1}|\widehat{\alpha}|+c_{2}|\widehat{\beta}|\right)}, \quad|G(\widehat{\alpha})| \leq C_{\varepsilon} e^{-2 \pi\left(c_{1}|\widehat{\alpha}|\right)},
$$

where

$$
\begin{aligned}
& c_{1}= \begin{cases}\|D\|^{-1}-\varepsilon, & \text { if } \widehat{\alpha}<0, \\
\text { arbitrarily large, } & \text { if } \widehat{\alpha}>0,\end{cases} \\
& c_{2}= \begin{cases}v D v^{t}-\varepsilon, & \text { if } \widehat{\beta}<0, \\
\text { arbitrarily large, } & \text { if } \widehat{\beta}>0 .\end{cases}
\end{aligned}
$$

Proof. Let $\alpha, \beta \in \mathcal{Q}$, with $\alpha=\alpha_{1}+i \alpha_{2}$ and $\beta=\beta_{1}+i \beta_{2}$. By Cauchy's formula,

$$
\begin{aligned}
& F(\widehat{\alpha}, \widehat{\beta})= \int_{-\infty+i \alpha_{1}}^{+\infty+i \alpha_{2}} \int_{-\infty+i \beta_{1}}^{+\infty+i \beta_{2}} e^{-2 \pi i(\alpha \widehat{\alpha}+\beta \widehat{\beta})} \frac{\partial f}{\partial \beta}(\alpha, \beta) d \alpha d \beta \\
&=e^{2 \pi\left(\alpha_{2} \widehat{\alpha}+\beta_{2} \widehat{\beta}\right)} \int_{-\infty}^{+\infty} \int_{-\infty}^{+\infty} e^{-2 \pi i\left(\alpha_{1} \widehat{\alpha}+\beta_{1} \widehat{\beta}\right)} \\
& \cdot \frac{\partial f}{\partial \beta}\left(\alpha_{1}+i \alpha_{2}, \beta_{1}+i \beta_{2}\right) d \alpha_{1} d \beta_{1} .
\end{aligned}
$$

Therefore,

$$
|F(\widehat{\alpha}, \widehat{\beta})| \leq e^{2 \pi\left(\alpha_{2} \widehat{\alpha}+\beta_{2} \widehat{\beta}\right)} \int_{\mathbb{R}^{2}}\left|\frac{\partial f}{\partial \beta}\left(\alpha_{1}+i \alpha_{2}, \beta_{1}+i \beta_{2}\right)\right| d \alpha_{1} d \beta_{1} .
$$

If $\widehat{\alpha}<0$, we use Lemma 6 , with any $0 \leq \alpha_{2}<\|D\|^{-1}$. If $\widehat{\alpha}>0$, we can use the previous argument with any negative $\alpha_{2}$. Same thing for $\beta$. Same thing for $G$.

Lemma 8. $G(\widehat{\alpha})=0$ when $\widehat{\alpha} \geq 0$. Also,

$$
G(\widehat{\alpha})=\frac{(-2 \widehat{\alpha})^{d / 2-1}}{\sqrt{\operatorname{det} \mathbb{D}}} \int_{\mathbf{n} \in S_{d-1}} \exp \left(2 \pi \widehat{\alpha}\left(\mathbf{n}, \mathbb{D}^{-1} \mathbf{n}\right)\right) d \sigma, \quad \widehat{\alpha}<0
$$

where $d \sigma$ denotes the usual surface measure on $S_{d-1}$. 
Proof. First $G$. Let $\phi \in C_{0}^{\infty}$

$$
\begin{aligned}
I & \equiv \int_{\mathbb{R}} G(\widehat{\alpha}) \phi(\widehat{\alpha}) d \widehat{\alpha} \\
& =\int_{\mathbb{R}} \int_{\mathbb{R}^{2}} e^{-2 \pi i \alpha \widehat{\alpha}} f(\alpha, 0) d \alpha d \widehat{\alpha} \\
& =\int_{\mathbb{R}} \int_{\mathbb{R}^{2}} \int_{\mathbb{R}^{d}} e^{-\pi|\xi|^{2}} e^{-2 \pi i \alpha \widehat{\alpha}} e^{-\pi i \alpha \xi \mathbb{D} \xi^{t}} \phi(\widehat{\alpha}) d \xi d \alpha d \widehat{\alpha} \\
& =\int_{\mathbb{R}^{2}} \int_{\mathbb{R}^{d}} e^{-\pi|\xi|^{2}} e^{-\pi i \alpha \xi \mathbb{D} \xi^{t}} \widehat{\phi}(\alpha) d \xi d \alpha \\
& =\int_{\mathbb{R}^{d}} e^{-\pi|\xi|^{2}} \phi\left(-\frac{1}{2} \xi \mathbb{D} \xi^{t}\right) d \xi .
\end{aligned}
$$

The substitution $\eta=\xi \mathbb{D}^{1 / 2}$ leads to

$$
\begin{aligned}
I & =\frac{1}{\sqrt{\operatorname{det} \mathbb{D}}} \int e^{-\pi\left(\eta, \mathbb{D}^{-1} \eta\right)} \phi\left(-\frac{1}{2}|\eta|^{2}\right) d \eta \\
& =\frac{1}{\sqrt{\operatorname{det} \mathbb{D}}} \int_{0}^{\infty} \int_{\mathbf{n} \in S_{d-1}} d o(\mathbf{n}) e^{-\pi r^{2}\left(\mathbf{n}, \mathbb{D}^{-1} \mathbf{n}\right)} \phi\left(-\frac{1}{2} r^{2}\right) r^{d-1} d r .
\end{aligned}
$$

By substituting $r$ with $\alpha=r^{2} / 2$, we find that

$$
I=\frac{1}{\sqrt{\operatorname{det} \mathbb{D}}} \int_{0}^{\infty} \int_{S_{d-1}} e^{-2 \pi \alpha\left(\mathbf{n}, \mathbb{D}^{-1} \mathbf{n}\right)} \phi(-\widehat{\alpha})(2 \widehat{\alpha})^{(d-1) / 2} d \sigma(\mathbf{n}) \frac{d \widehat{\alpha}}{\sqrt{2 \widehat{\alpha}}} .
$$

Let us introduce the polar coordinate in the $(\widehat{\alpha}, \widehat{\beta})$ plane

$$
\widehat{\rho}=\sqrt{\widehat{\alpha}^{2}+\widehat{\beta}^{2}}, \quad \widehat{\theta}=\pi+\tan ^{-1}\left(\frac{\widehat{\beta}}{\widehat{\alpha}}\right),
$$

where the arc-tangent is taken with values in $(-\pi / 2, \pi / 2)$ and the angle $\widehat{\theta}$ is defined with a shift of $\pi$ to keep notation simple in what follows.

Lemma 9. The function $F(\widehat{\alpha}, \widehat{\beta})$ has support in the sector

$$
0 \leq \widehat{\theta} \leq \tan ^{-1}\|v\|^{2}
$$

Inside this sector, we have that

$$
F(\widehat{\rho}, \widehat{\theta})=-\frac{4 \pi i}{\sqrt{\operatorname{det} \mathbb{D}}} \widehat{\rho}^{d}(2 \cos \widehat{\theta})^{d-1} \sqrt{\frac{\tan \widehat{\theta}}{\|v\|^{2}-\tan \hat{\theta}}} m(\widehat{\rho}, \widehat{\theta}),
$$


where

$$
m(\widehat{\theta}, \widehat{\rho})=\int_{\Gamma_{\widehat{\theta}}} e^{-2 \pi \widehat{\rho} \cos \widehat{\theta}\left(\mathbf{n}, \mathbb{D}^{-1} \mathbf{n}\right)} d \sigma_{\Gamma_{\widehat{\theta}}},
$$

$\Gamma_{\widehat{\theta}}$ is the sphere

$$
\Gamma_{\widehat{\theta}}=\left\{\mathbf{n}:\|\mathbf{n}\|^{2}=1 \text { and } v \cdot \mathbf{n}=\sqrt{\tan \widehat{\theta}}\right\}
$$

and $d \sigma_{\Gamma_{\widehat{\theta}}}$ is the surface measure on $\Gamma_{\theta}$.

Proof. Denote $w=\sqrt{\mathbb{D}} v$.

$$
\begin{aligned}
& I \equiv \iint F(\widehat{\alpha}, \widehat{\beta}) \phi(\widehat{\alpha}, \widehat{\beta}) d \widehat{\alpha} d \widehat{\beta} \\
&=-\pi i \int(\xi \cdot w)^{2} e^{-\pi\left|\xi^{2}\right|} e^{-2 \pi i(\alpha \widehat{\alpha}+\beta \widehat{\beta})} e^{-\pi i \xi\left(\alpha \mathbb{D}+\beta w^{t} w\right) \xi^{t}} \\
& \\
& \quad \cdot \phi(\widehat{\alpha}, \widehat{\beta}) d \widehat{\alpha} d \widehat{\beta} d \alpha d \beta d \xi \\
&=-\pi i \int(\xi \cdot w)^{2} e^{-\pi\left|\xi^{2}\right|} e^{-\pi i \xi\left(\alpha \mathbb{D}+\beta w^{t} w\right) \xi^{t}} \widehat{\phi}(\alpha, \beta) d \alpha d \beta d \xi \\
&=-\pi i \int(\xi \cdot w)^{2} e^{-\pi\left|\xi^{2}\right|} \phi\left(-\frac{1}{2} \xi \mathbb{D} \xi^{t},-\frac{1}{2}(w \cdot \xi)^{2}\right) d \xi
\end{aligned}
$$

In terms of the new coordinate $\eta=\xi \mathbb{D}^{1 / 2}$, we find that

$$
\begin{gathered}
I=-\frac{\pi i}{\sqrt{\operatorname{det} \mathbb{D}}} \int_{\mathbb{R}^{d}}(v \cdot \eta)^{2} e^{-\pi\left(\eta, \mathbb{D}^{-1} \eta\right)} \phi\left(-\frac{1}{2} \eta^{2},-\frac{1}{2}(v \cdot \eta)^{2}\right) \\
=-\frac{\pi i}{\sqrt{\operatorname{det} \mathbb{D}}} \int_{0}^{\infty} d r \int_{\mathbf{n} \in S_{d-1}} d \sigma(\mathbf{n}) r^{d+1}(v \cdot \mathbf{n})^{2} e^{-\pi r^{2}\left(\mathbf{n}, \mathbb{D}^{-1} \mathbf{n}\right)} \\
\cdot \phi\left(-\frac{1}{2} r^{2},-\frac{1}{2} r^{2}(v \cdot \mathbf{n})^{2}\right) .
\end{gathered}
$$

In polar coordinates,

$$
\widehat{\rho}=\frac{1}{2} r^{2} \sqrt{1+(v \cdot \mathbf{n})^{2}}, \quad \widehat{\theta}=\tan ^{-1}(v \cdot \mathbf{n})^{2},
$$

and the function

$$
\psi(\widehat{\rho}, \widehat{\theta})=\phi(\widehat{\alpha}(\widehat{\rho}, \widehat{\theta}), \widehat{\beta}(\widehat{\rho}, \widehat{\theta}))
$$


We have that

$$
\begin{gathered}
I=-\frac{\pi i}{\sqrt{\operatorname{det} \mathbb{D}}} \int_{0}^{\infty} d r \int_{\mathbf{n} \in S_{d-1}} d \sigma(\mathbf{n}) r^{d+1}(v \cdot \mathbf{n})^{2} e^{-\pi r^{2}\left(\mathbf{n}, \mathbb{D}^{-1} \mathbf{n}\right)} \\
\cdot \psi\left(\widehat{\rho}, \tan ^{-1}(v \cdot \mathbf{n})^{2}\right) .
\end{gathered}
$$

The unit sphere $S_{d-1}=\left\{\mathbf{n}:|\mathbf{n}|^{2}=1\right\}$ intersects the plane of equation $v \cdot \mathbf{n}=\sqrt{\tan \widehat{\theta}}$ on a codimension 2 sphere $\Gamma_{\widehat{\theta}}$, (which might degenerate to a point or be empty). The points of $\Gamma_{\widehat{\theta}}$ have the form

$$
\mathbf{n}=\sqrt{\tan \widehat{\theta}} \frac{v}{|v|^{2}}+\zeta
$$

with

$$
\zeta \cdot v=0
$$

Hence, the radius is

$$
\sqrt{\left(1-\frac{\tan \hat{\theta}}{\|v\|^{2}}\right)_{+}}
$$

and the $(d-2)$-dimensional volume of such set is

$$
\frac{2 \pi^{d / 2-1}}{(d-2) \Gamma\left(\frac{d}{2}-1\right)}\left(1-\frac{\tan \widehat{\theta}}{\|v\|^{2}}\right)_{+}^{d / 2-1} .
$$

The co-area formula (see Chavel [Cha]) for spheres reads as follows

$$
\int_{\mathbf{n} \in S_{d-1}} f(\mathbf{n}) d \sigma_{d-1}=\int_{-1}^{1} \int_{\substack{\mathbf{n} \cdot v_{0}=t \\ \mathbf{n} \in S_{d-1}}} f(\mathbf{n}) d A_{t} \frac{d t}{\sqrt{1-t^{2}}}, \quad v_{0} \in S_{d-1}
$$

where $d A_{t}$ is the $(d-2)$-dimensional surface measure on that sphere. In our context, $v_{0}=v /\|v\|$ and this formula implies that

$$
\int_{\mathbf{n} \in S_{d-1}} f(\mathbf{n}) d \sigma_{d-1}=\int_{0}^{\|v\|} \frac{d \sqrt{\tan \widehat{\theta}}}{\sqrt{\|v\|^{2}-\tan \widehat{\theta}}} \int_{\Gamma_{\widehat{\theta}}} f(\mathbf{n}) d \sigma_{\Gamma_{\widehat{\theta}}}
$$


Thus,

$$
\begin{aligned}
& I=-\frac{\pi i}{\sqrt{\operatorname{det} \mathbb{D}}} \int_{0}^{\infty} d r r^{d+1} \int_{0}^{\|v\|} \tan \widehat{\theta} \frac{d \sqrt{\tan \hat{\theta}}}{\sqrt{\|v\|^{2}-\tan \hat{\theta}}} \\
& \cdot \psi(\widehat{\rho}, \widehat{\theta}) \int_{\mathbf{n} \in \Gamma_{\widehat{\theta}}} e^{-\pi r^{2}\left(\mathbf{n}, \mathbb{D}^{-1} \mathbf{n}\right)} d \Gamma_{\widehat{\theta}} \\
& =-\frac{\pi i}{\sqrt{\operatorname{det} \mathbb{D}}} \int_{0}^{\infty} d \widehat{\rho} \int_{0}^{\|v\|} \frac{d \sqrt{\tan \widehat{\theta}}}{\sqrt{\|v\|^{2}-\tan \widehat{\theta}}} \\
& \text { - }(2 \widehat{\rho} \cos \widehat{\theta})^{d+1} \tan \widehat{\theta} m(\widehat{\rho}, \widehat{\theta}) \psi(\widehat{\rho}, \widehat{\theta}) \\
& =-\frac{\pi i}{\sqrt{\operatorname{det} \mathbb{D}}} \int_{0}^{\infty} d \widehat{\rho} \int_{0}^{\tan ^{-1}\|v\|^{2}} d \widehat{\theta} \frac{(2 \widehat{\rho} \cos \widehat{\theta})^{d+1} \sqrt{\tan \widehat{\theta}}}{\cos ^{2} \widehat{\theta} \sqrt{\|v\|^{2}-\tan \hat{\theta}}} \\
& \text { - } m(\widehat{\rho}, \widehat{\theta}) \psi(\widehat{\rho}, \widehat{\theta})
\end{aligned}
$$

where $m(\widehat{\theta}, \widehat{\rho})$ is the function defined in the statement of the lemma. Since

$$
I=\int_{0}^{\infty} \int_{-\pi}^{\pi} F(\widehat{\rho}, \widehat{\theta}) \psi(\widehat{\rho}, \widehat{\theta}) \widehat{\rho} d \widehat{\rho} d \widehat{\theta}
$$

we conclude that $F$ is supported on the sector

$$
\operatorname{supp} F=\left\{(\widehat{\rho}, \widehat{\theta}):|\widehat{\theta}| \leq \tan ^{-1}\|v\|^{2}\right\},
$$

and

$$
F(\widehat{\rho}, \widehat{\theta})=-\frac{4 \pi i}{\sqrt{\operatorname{det} \mathbb{D}}} \widehat{\rho}^{d}(2 \cos \widehat{\theta})^{d-1} \sqrt{\frac{\tan \hat{\theta}}{\|v\|^{2}-\tan \hat{\theta}}} m(\widehat{\rho}, \widehat{\theta})
$$

\section{Lemma 11.}

$$
\begin{aligned}
I\left(R^{2}\right)=R^{d / 2}\left(\int_{-\infty}^{0} d \widehat{\alpha} \frac{J_{d / 2}(2 R \pi \sqrt{|\widehat{\alpha}|}) G(\widehat{\alpha})}{(2|\widehat{\alpha}|)^{d / 4}}\right. \\
+\pi i \int_{-\infty}^{0} d \widehat{\alpha} \int_{\widehat{\alpha} \tan ^{-1}\|v\|^{2}}^{0} d \widehat{\beta} \frac{J_{d / 2}(2 R \pi \sqrt{|\widehat{\alpha}|}) F(\widehat{\alpha}, \widehat{\beta})}{(2|\widehat{\alpha}|)^{d / 4}} \\
\left.\cdot \frac{\cos (2 \pi \sqrt{2 \widehat{\beta}}-1}{2 \pi^{2} \widehat{\beta}}\right) .
\end{aligned}
$$


Proof. Lemma 7 allows us to continue Lemma 2 as

$$
\begin{aligned}
& I\left(R^{2}\right)=\pi^{d / 2} \sum_{k} R^{d+2 k}(\pi i)^{k} a_{k, 0} \int_{\mathbb{R}}(2 \pi i \widehat{\alpha})^{k} G(\widehat{\alpha}) d \widehat{\alpha} \\
& +\pi^{d / 2} \sum_{k, j} R^{d+2 k}(\pi i)^{k+j+1} a_{k, j+1} \\
& \cdot \int_{\mathbb{R}^{2}}(2 \pi i \widehat{\alpha})^{k}(2 \pi i \widehat{\beta})^{j} F(\widehat{\alpha}, \widehat{\beta}) d \widehat{\alpha} d \widehat{\beta} . \\
& =\pi^{d / 2} \int_{\mathbb{R}} \sum_{k} R^{d+2 k}\left(-2 \pi^{2}\right)^{k} a_{k, 0} \widehat{\alpha}^{k} G(\widehat{\alpha}) d \widehat{\alpha} \\
& +\pi^{d / 2} \int_{\mathbb{R}^{2}} \sum_{k, j} R^{d+2 k}\left(-2 \pi^{2}\right)^{k+j+1} a_{k, j+1} \widehat{\alpha}^{k} \widehat{\beta}^{j} F(\widehat{\alpha}, \widehat{\beta}) d \widehat{\alpha} d \widehat{\beta} \\
& =R^{d} \pi^{d / 2} \int_{\mathbb{R}} \sum_{k}\left(-2 R^{2} \pi^{2} \widehat{\alpha}\right)^{k} a_{k, 0} F(\widehat{\alpha}, \widehat{\beta}) d \widehat{\alpha} \\
& +R^{d} \pi^{d / 2} \int_{\mathbb{R}^{2}} \sum_{k, j}\left(-2 R^{2} \pi^{2} \widehat{\alpha}\right)^{k}\left(-2 \pi^{2} \widehat{\beta}\right)^{j} a_{k, j+1} F(\widehat{\alpha}, \widehat{\beta}) d \widehat{\alpha} d \widehat{\beta} \\
& =R^{d} \pi^{d / 2} \int_{\mathbb{R}} \sum_{k, 0}\left(2 R^{2} \pi^{2} \widehat{\alpha}\right)^{k}\left|a_{k, 0}\right| G(\widehat{\alpha}) d \widehat{\alpha} \\
& +R^{d} \pi^{d / 2} \int_{\mathbb{R}^{2}} \sum_{k, j}\left(2 R^{2} \pi^{2} \widehat{\alpha}\right)^{k}\left(2 \pi^{2} \widehat{\beta}\right)^{j}\left|a_{k, j+1}\right| F(\widehat{\alpha}, \widehat{\beta}) d \widehat{\alpha} d \widehat{\beta} . \\
& =R^{d} \pi^{d / 2}\left(\int_{\mathbb{R}} \mathcal{Z}\left(4 R^{2} \pi^{2} \widehat{\alpha}\right) G(\widehat{\alpha}) d \widehat{\alpha}\right. \\
& \left.+\int_{\mathbb{R}^{2}} \widetilde{\mathcal{B}}\left(4 R^{2} \pi^{2} \widehat{\alpha}, 4 \pi^{2} \widehat{\beta}\right) F(\widehat{\alpha}, \widehat{\beta}) d \widehat{\alpha} d \widehat{\beta}\right)
\end{aligned}
$$

and the last integral converges unconditionally due to the growth properties of $\mathcal{B}$, in conjunction with Lemma 7 .

Lemma 12. We have that

$$
I\left(R^{2}\right)=R^{d / 2} \sum_{j=0}^{\infty} \frac{1}{j !} \int_{-\infty}^{0} \frac{J_{d / 2}(2 R \pi \sqrt{|\widehat{\alpha}|})}{(2|\widehat{\alpha}|)^{d / 4}} H^{(j)}(\widehat{\alpha}) d \widehat{\alpha}
$$


where

$$
\begin{aligned}
H^{(j)}(\widehat{\alpha})= & \frac{(2 \pi)^{2 j}(-2 \widehat{\alpha})^{(d+j) / 2-1}}{\sqrt{\operatorname{det} \mathbb{D}}} \\
& \cdot \int_{\mathbf{n} \in S_{d-1}}(\mathbf{n} \cdot v)^{j} \exp \left(2 \pi \widehat{\alpha}\left(\mathbf{n}, \mathbb{D}^{-1} \mathbf{n}\right)\right) d \sigma(\mathbf{n}),
\end{aligned}
$$

with $\widehat{\alpha}<0$.

Proof. We have that

$$
H^{(j)}(\widehat{\alpha})=i 2^{3 j-1} \pi^{2 j-1} \int_{\widehat{\alpha} \tan ^{-1}\|v\|^{2}}^{0} d \widehat{\beta} F(\widehat{\alpha}, \widehat{\beta}) \widehat{\beta}^{j-1} .
$$

Hence

$$
I_{j} \equiv \int_{-\infty}^{\infty} d \widehat{\alpha} H^{(j)}(\widehat{\alpha}) \phi(\widehat{\alpha})=(2 \pi)^{2 j} \int d \xi(\xi \cdot w)^{2 j} e^{-\pi\left|\xi^{2}\right|} \phi\left(-\frac{1}{2} \xi \mathbb{D} \xi^{t}\right) .
$$

where $w=\sqrt{\mathbb{D}} v$. Proceeding as in the proof of Lemma 10, we make the substitution $\eta=\sqrt{\mathbb{D}} \xi$ and obtain

$$
\begin{aligned}
I_{j}= & \frac{(2 \pi)^{2 j}}{\sqrt{\operatorname{det} \mathbb{D}}} \int(\eta \cdot v)^{j} e^{-\pi\left(\eta, \mathbb{D}^{-1} \eta\right)} \phi\left(-\frac{1}{2}|\eta|^{2}\right) d \eta \\
= & \frac{(2 \pi)^{2 j}}{\sqrt{\operatorname{det} \mathbb{D}}} \int_{0}^{\infty} r^{j+d-1} d r \int_{\mathbf{n} \in S_{d-1}} d \sigma(\mathbf{n})(\mathbf{n} \cdot v)^{j} \\
\cdot & e^{-\pi r^{2}\left(\mathbf{n}, \mathbb{D}^{-1} \mathbf{n}\right)} \phi\left(-\frac{1}{2} r^{2}\right) .
\end{aligned}
$$

By substituting $r$ with $\alpha=r^{2} / 2$, we find that

$$
\begin{aligned}
I_{j}=\frac{(2 \pi)^{2 j}}{\sqrt{\operatorname{det} \mathbb{D}} \int_{0}^{\infty} \int_{S_{d-1}}}(\mathbf{n} \cdot v)^{j} e^{-2 \pi \alpha\left(\mathbf{n}, \mathbb{D}^{-1} \mathbf{n}\right)} \\
\quad \cdot \phi(-\widehat{\alpha})(2 \widehat{\alpha})^{(j+d-1) / 2} \frac{d \widehat{\alpha}}{\sqrt{2 \widehat{\alpha}}} d \sigma(\mathbf{n}) .
\end{aligned}
$$




\section{The hyperbolic case.}

The hyperbolic case can be reduced to the positive definite case, via spherical convolutions.

We will use the following elementary identities

$$
\frac{d}{d r} \int_{|x| \leq r} f(x) d x=\int_{|x|=r} f(\sigma) d \sigma
$$

and

$$
\iint_{|x|^{2}-|y|^{2} \leq R} \varphi(x, y) d x d y=\int_{0}^{\infty} \int_{|y|=r} \int_{|x| \leq R+r^{2}} \varphi(x, y) d x d \sigma(y) d r .
$$

We apply these identities to $I$ with the notation of Lemma 1 (namely $n=n_{1}+n_{2}$ ), to obtain

$$
\begin{aligned}
I(K) & =\int_{\left|x-v_{1}\right|^{2}-\left|y-v_{2}\right|^{2} \leq K} e^{-\left(x, \mathbb{D}_{1} x\right)-\left(y, \mathbb{D}_{2} y\right)} \\
& =\int_{0}^{\infty} \int_{\left|y-v_{2}\right|^{2}=r^{2}} e^{-\left(y, \mathbb{D}_{2} y\right)} \int_{\left|x-v_{1}\right|^{2} \leq r^{2}+K} e^{-\left(x, \mathbb{D}_{1} x\right)} d x d \sigma(y) d r \\
& =\int_{0}^{\infty} I_{1}\left(r^{2}+K\right) \frac{\partial}{\partial r} I_{2}\left(r^{2}\right) d r
\end{aligned}
$$

Here, the integrals $I_{1}$ and $I_{2}$ are both of the positive definite type, with matrices $\mathbb{D}_{1}$ and $\mathbb{D}_{2}$ respectively, and offset vectors $v_{1}$ and $v_{2}$, in dimension $n_{1}$ and $n_{2}$. We can therefore deal with the methods in the preceeding section. In particular, let $H_{1}^{(j)}(\widehat{\alpha})$ and $H_{2}^{(j)}(\widehat{\alpha})$ be the functions associated to the integrals $I_{1}$ and $I_{2}$, respectively, and let

$$
H_{i}(\widehat{\alpha})=\sum_{j=0}^{\infty} \frac{1}{j !} H_{i}^{(j)}(\widehat{\alpha})
$$

where $i=1,2$, so that

$$
I_{i}\left(R^{2}\right)=R^{n_{i} / 2} \int_{-\infty}^{0} \frac{J_{n_{1} / 2}(2 \pi R \sqrt{|\widehat{\alpha}|})}{(2|\widehat{\alpha}|)^{n_{i} / 4}} H(\widehat{\alpha}) d \widehat{\alpha}
$$


Equation (5.1) then implies that

$$
\begin{aligned}
I(K)= & \int_{0}^{\infty} I_{1}\left(r^{2}+K\right) \frac{\partial}{\partial r} I_{2}(r) d r \\
= & \int_{0}^{\infty} \iint_{\mathbb{R}_{-}^{2}}\left(r^{2}+K\right)^{n_{1} / 4} \frac{J_{n_{1} / 2}\left(2 \pi \sqrt{\left(r^{2}+K\right)\left|\widehat{\alpha}_{1}\right|}\right)}{\left(2\left|\widehat{\alpha}_{1}\right|\right)^{n_{1} / 4}\left(2\left|\widehat{\alpha}_{2}\right|\right)^{n_{2} / 4}} \\
& \cdot \partial_{r}\left(r^{n_{2} / 2} J_{n_{2} / 2}\left(2 \pi r \sqrt{\left|\widehat{\alpha}_{2}\right|}\right)\right) H\left(\widehat{\alpha}_{1}\right) H_{2}\left(\widehat{\alpha}_{2}\right) d \widehat{\alpha}_{1} d \widehat{\alpha}_{2} \\
= & 2^{-n / 4} \int \Theta\left(\widehat{\alpha}_{1}, \widehat{\alpha}_{2}\right) H_{1}\left(\widehat{\alpha}_{1}\right) H_{2}\left(\widehat{\alpha}_{2}\right) d \widehat{\alpha}_{1} d \widehat{\alpha}_{2},
\end{aligned}
$$

where

$$
\begin{gathered}
\Theta\left(\widehat{\alpha}_{1}, \widehat{\alpha}_{2}\right)=\int_{0}^{\infty}\left(r^{2}+K\right)^{n_{1} / 4} \frac{J_{n_{1} / 2}\left(2 \pi \sqrt{\left(r^{2}+K\right)\left|\widehat{\alpha}_{1}\right|}\right)}{\left|\widehat{\alpha}_{1}\right|^{n_{1} / 4}\left|\widehat{\alpha}_{2}\right|^{n_{2} / 4}} \\
\cdot \partial_{r}\left(r^{n_{2} / 2} J_{n_{2} / 2}\left(2 \pi r \sqrt{\left|\widehat{\alpha}_{2}\right|}\right)\right)
\end{gathered}
$$

\section{Feynmann expansion for moments.}

It will suffice to consider the positive definite case. To compute the functions $H^{(j)}(\widehat{\alpha})$ one can make use of the following ansätze which satisfy the asymptotic properties of the exact functions

$$
H^{(j)}(\widehat{\alpha}) \approx(-2 \widehat{\alpha})^{d / 2-1} P^{(j)}(\widehat{\alpha}) e^{-m \widehat{\alpha}},
$$

where $m=2 \pi \inf \sigma\left(\mathbb{D}^{-1}\right)$ and the $P^{(j)}(\widehat{\alpha})$ are polynomials of the form

$$
P^{(j)}(\widehat{\alpha})=\sum_{k=0}^{n} c_{k}^{(j)} \widehat{\alpha}^{j}
$$

To estimate the coefficients in the polynomials, one can match moments.

Let

$$
\mathbb{B}=i \alpha \mathbb{D}+i \beta\|v\|^{2} \mathbb{D}^{1 / 2} \mathbb{P}_{v} \mathbb{D}^{1 / 2}
$$


To compute the determinant $\operatorname{det}(1+\mathbb{B})$, we can use a Feynmann expansion.

$$
\begin{aligned}
\operatorname{det}(1+\mathbb{B})= & \exp (\operatorname{Tr} \log (1+\mathbb{B})) \\
= & \exp \left(\operatorname{Tr} \sum_{k=1}^{\infty} \frac{(-1)^{k-1}}{k} \mathbb{B}^{k}\right) \\
= & \exp \left(\sum_{k=1}^{\infty} \frac{(-1)^{k-1}}{k} \operatorname{Tr} \mathbb{B}^{k}\right) \\
= & \sum_{n=0}^{\infty} \frac{1}{n !}\left(\sum_{k=1}^{\infty} \frac{(-1)^{k-1}}{k} \operatorname{Tr} \mathbb{B}^{k}\right)^{n} \\
= & 1+\operatorname{Tr} \mathbb{B}+\left(-\frac{1}{2} \operatorname{Tr} \mathbb{B}^{2}+\frac{1}{2}(\operatorname{Tr} \mathbb{B})^{2}\right) \\
& +\left(\frac{2}{3} \operatorname{Tr} \mathbb{B}^{3}-(\operatorname{Tr} \mathbb{B})\left(\operatorname{Tr} \mathbb{B}^{2}\right)+\frac{1}{3}(\operatorname{Tr} \mathbb{B})^{3}\right) \\
& +\left(-\frac{3}{2} \operatorname{Tr} \mathbb{B}^{4}+\frac{3}{4}\left(\operatorname{Tr} \mathbb{B}^{2}\right)^{2}+2 \operatorname{Tr} \mathbb{B}^{T r} \mathbb{B}^{3}\right. \\
& \left.\quad-\frac{3}{2}(\operatorname{Tr} \mathbb{B})^{2} \operatorname{Tr} \mathbb{B}^{2}+\frac{1}{4}(\operatorname{Tr} \mathbb{B})\right) \\
& +\cdots
\end{aligned}
$$

Traces of powers of $\mathbb{B}$ are given by

$$
\operatorname{Tr}\left(\mathbb{B}^{k}\right)=i^{k}\left(\frac{\left(v, \mathbb{D}^{k} v\right)}{\|v\|^{2}}\left(\left(\alpha+\beta\|v\|^{2}\right)^{k}-\alpha^{k}\right)+\alpha^{k} \operatorname{Tr} \mathbb{D}^{k}\right),
$$

where we use the fact that the projection operator $\mathbb{P}_{v}$ is nilpotent.

Here are some moments computed by means of this formula

$$
\begin{gathered}
\int d \widehat{\alpha} H^{(0)}(\widehat{\alpha})=1 \\
\int d \widehat{\alpha} \widehat{\alpha} H^{(0)}(\widehat{\alpha})=-\frac{1}{2} \operatorname{Tr} \mathbb{D} \\
\int d \widehat{\alpha} \widehat{\alpha}^{2} H^{(0)}(\widehat{\alpha})=\frac{1}{2} \operatorname{Tr} \mathbb{D}^{2}+\frac{1}{4}(\operatorname{Tr} \mathbb{D})^{2}, \\
\int d \widehat{\alpha} H^{(1)}(\widehat{\alpha})=-\frac{1}{2} \operatorname{Tr} \mathbb{D},
\end{gathered}
$$




$$
\begin{aligned}
& \int d \widehat{\alpha} \widehat{\alpha} H^{(1)}(\widehat{\alpha})=\frac{1}{4} \operatorname{Tr} \mathbb{D}(v, \mathbb{D} v)+\frac{1}{2}\left(v, \mathbb{D}^{2} v\right) \\
& \int d \widehat{\alpha} \widehat{\alpha}^{2} H^{(1)}(\widehat{\alpha})=-\frac{19}{8} \operatorname{Tr} \mathbb{D}(v, \mathbb{D} v)^{2}-\frac{3}{2} \frac{\left.\left(v, \mathbb{D}^{2} v\right)-\operatorname{Tr} \mathbb{D}(v, \mathbb{D} v)\right)}{(v, \mathbb{D} v)} \\
&-\frac{3}{4} \operatorname{Tr} \mathbb{D}\left(\left(v, \mathbb{D}^{2} v\right)\|v\|-(v, \mathbb{D} v)^{2}\right) \\
&+\frac{1}{2}\left(v, \mathbb{D}^{2} v\right)\|v\| \operatorname{Tr} \mathbb{D}+(v, \mathbb{D} v)\left(v, \mathbb{D}^{2} v\right) \\
&-\left(v, \mathbb{D}^{3} v\right)\|v\|
\end{aligned}
$$

The coefficients $c_{k}^{j}$ in (6.2) can be computed by matching the momenta above. This involves solving a linear system. In fact, based on the ansatz in (6.1) we have that

$$
\begin{aligned}
\int d \widehat{\alpha} \widehat{\alpha}^{n} H^{(j)}(\widehat{\alpha}) & \approx \int d \widehat{\alpha}(-2 \widehat{\alpha})^{d / 2-1} P^{(j)}(\widehat{\alpha}) e^{m \widehat{\alpha}} \\
& =\sum_{k=0}^{n} c_{k}^{(j)} \int d \widehat{\alpha}(-2 \widehat{\alpha})^{d / 2-1} \widehat{\alpha}^{j} e^{m \widehat{\alpha}} \\
& =-\sum_{k=0}^{n} c_{k}^{(j)} 2^{d / 2-1} m^{-d / 2-j} \int_{0}^{\infty} d x x^{j+d / 2-1} e^{-x} \\
& =-\sum_{k=0}^{n} c_{k}^{(j)} 2^{d / 2-1} \Gamma\left(j+\frac{d}{2}\right) m^{-d / 2-j}
\end{aligned}
$$

\section{Conclusions.}

In this article, we develop a resummed perturbation expansion for the calculation of high dimensional Gaussian integrals on sets bounded by quadrics. Such integrals arise in the calculation of Value at Risk for large portfolios in the quadratic approximation.

After an initial simultaneous diagonalization of the covariance matrix and of the total gamma of the portfolio, we reduce the calculation of the Value at Risk to an integral of a Gaussian over a high dimensional quadric. This integral is computed using techniques from harmonic analysis, which reduce all calculations to the Fourier transform of quadrics. In the case of positive definite Gammas, this reduces to 
explicit Bessel functions; the general case is not much more difficult. The asymptotic behaviour of the relevant functions can be computed analytically up to factors which are smooth and bounded. The moments of these transforms can be computed by means of a Feynmann expansion and can be expressed in terms of linear invariants such as traces and determinants. This yields analytic formulas for the Value at Risk as a function of the time horizon.

Possible applications of this Fourier transform method that we can envisage include:

i) Performing real time monitoring of Value at Risk.

ii) Finding the impact of the sale of one single contract to the global risk exposure in real time, thus permitting to price against the current holdings.

iii) Identifying the risk factors which are mostly responsible for large Value at Risk.

iv) Visualizing and monitoring the risk exposure in terms of few parameters. (The Fourier transform we compute captures all the risk exposure effects in just two variables and contains information about the interplay between delta risk and gamma risk.)

v) Estimating "Bayesian" Value at Risk by integrating the covariance matrix over the Wishart distribution.

vi) VaR calibration from historical P\&L data.

These applications and extensions will be covered in forthcoming papers.

Acknowledgements. This research was partially supported by a grant from Algorithmics Inc. L. S. is partially supported by NSERC and the Sloan Foundation.

\section{References.}

[Cha] Chavel, I., Eigenvalues in Riemannian Geometry. Academic Pres, 1984.

[JPM] J. P. Morgan staff, RiskMetrics Technical Document. Third Edition, 1995. 
[KJB] Kotz, S., Johnson, N. L., Boyd, D. W., Distribution of quadratic forms, I and II. Ann. Math. Statist. 38 (1995), 823-848.

[Q] Quintanilla, M., An Asymptotic Expansion for Value at Risk. M. Sc. Thesis, University of Toronto, 1997.

[Rub] Ruben, H., A new result in the distribution of quadratic forms. Ann. Math. Statist. 33 (1962), 542-570.

Recibido: 4 de febrero de 1.999

Claudio Albanese and Luis Seco

Department of Mathematics

University of Toronto

100 St. George Street

Toronto ON M5S 3G3, CANADA

albanese@math. toronto.edu

seco@math.toronto.edu 